\title{
The Cost-Effectiveness of Treatments in Non-Cirrhotic Saudi Arabian Patients with Genotype 1 and Genotype 4 Chronic Hepatitis C
}

\author{
Mohammad Alowairdhi ${ }^{1,2}$, Varun Vaidya ${ }^{3,4 *}$, Eric Sahloff ${ }^{4}$ and Cindy \\ Puffer $^{5}$ \\ ${ }^{1}$ Department of Health Outcomes and Socioeconomic Sciences, University of Toledo, \\ Toledo, OH, USA \\ ${ }^{2}$ Senior Pharmacist, Pricing and Pharmacoeconomics Department, Drug Sector, Saudi \\ Food and Drug Authority, USA \\ ${ }^{3}$ Division Head, Center for Pharmaceutical Care and Outcomes Research, College of \\ Pharmacy and Pharmaceutical Sciences, University of Toledo, Toledo, OH, USA \\ ${ }^{4}$ Associate Professor, Department of Health Outcomes and Socioeconomic Sciences, \\ University of Toledo, Toledo, $\mathrm{OH}, \mathrm{USA}$ \\ ${ }^{5}$ Managed Care Pharmacy Operations Manager, Department of Health Outcomes and \\ Socioeconomic Sciences, University of Toledo, Toledo, OH, USA \\ *Corresponding Author: Varun Vaidya, Division Head, Center for Pharmaceutical Care \\ and Outcomes Research, College of Pharmacy and Pharmaceutical Sciences and Associate \\ Professor, Department of Health Outcomes and Socioeconomic Sciences, University of \\ Toledo, Toledo, OH, USA.
}

Received: May 28, 2020

Published: August 26, 2020

(C) All rights are reserved by Mayadhar Barik., et al.

\section{Abstract}

Objectives: (1) To estimate the total costs of hepatitis $C$ treatment choices recommended by the Saudi Association for the Study of Liver Diseases and Transplantation (SASLT) based on data from the Saudi Food and Drug Authority (SFDA), (2) To develop and operationalize the decision tree model and calculate the base case incremental cost-effectiveness ratio (ICER), (3) To perform oneway and probabilistic sensitivity analyses testing the underlying assumptions in the decision tree model.

Methods: A cost-effectiveness analysis was performed on a hypothetical cohort comparing different chronic hepatitis $\mathrm{C}$ treatment strategies from the (SFDA)'s perspective over a three-month period using a decision tree model. Data for this study were obtained retrospectively from the (SFDA) and published literature. Costs were measured in United States Dollars (USD). Life-years gained (Ly) were the outcomes measured in this study. Since the SASLT guidelines differ between genotype 1 and genotype 4, There were two separate decision tree models and analyses for each genotype cohort at a willingness to pay (WTP) of $\$ 65,000$.

Result and Discussion: In genotype 1 base case analysis, the incremental cost-effectiveness comparison between the interventions showed that both Elbasvir/Grazoprevir and Paritaprevir/Ritonavir/Ombitasvir plus Dasabuvir with Ribavirin (3D+RBV) dominated Sofosbuvir with Simeprevir and Ledipasvir/Sofosbuvir. Against 3D+RBV, the ICER was \$33,796/Ly for each additional cure. In genotype 4 base case analysis, Paritaprevir/Ritonavir/Ombitasvir with Ribavirin (2D+RBV) dominated Ledipasvir/Sofosbuvir and Sofosbuvir plus Simeprevir. The interventions compared in genotype 1 are competitive and cost effective in exception of Sofosbuvir with Simeprevir while $2 \mathrm{D}+\mathrm{RBV}$ is highly recommended in genotype 4. Interventions in both genotypes will be dominated by Keywords: Hepatitis C; Genotype 1; Genotype 4

Citation: Varun Vaidya., et al. "The Cost-Effectiveness of Treatments in Non-Cirrhotic Saudi Arabian Patients with Genotype 1 and Genotype 4 Chronic Hepatitis C". Acta Scientific Medical Sciences 4.9 (2020): 03-14. 


\section{Introduction}

Hepatitis $\mathrm{C}$ is a viral infection carried by the blood stream which infects liver cells. After the replication of the virus, Ribonucleic Acid (RNA) within the infected liver cells continues to spread to other liver cells, causing hepatitis C [1]. The virus upon prolonged incubation beyond 24 weeks is considered a chronic infection [2]. The implications of this disease may lead to liver fibrosis, liver cirrhosis, liver cancer, or death. Hepatitis C virus (HCV) is a blood-borne virus that is transmitted via unsterile syringes or similar equipment which injects drugs into the blood stream or by blood donation [3]. With no vaccination developed for hepatitis $\mathrm{C}$ virus, the best way to prevent this infection is by avoiding these behaviors, using sterilization, or blood screening. Hepatitis $C$ virus infection through occupational, perinatal, and sexual contact happens less frequently than percutaneous exposures [3]. There are 7 genotypes of the hepatitis $C$ virus and more than 100 subtypes, and each has their characteristics and prevalence worldwide, all responding differently to treatment $[4,5]$. Furthermore, there are possibilities that one individual can be infected with more than one genotype [6]. The virus genotype and degree of liver damage are used to guide disease management and treatment decisions $[1,3]$.

Chronic Hepatitis C (CHC) is one of the world's major causes of liver disease and a potential cause of mortality and morbidity infecting between 130 - 175 million individuals and is responsible for 500,000 deaths each year $[7,8]$. Chronic hepatitis $C$ genotype prevalence differs depending on the region and geographical distribution of the population. Genotype 1 and genotype 4 are the most prevalent genotypes worldwide with approximately onethird of the cases occurring in East Asia and Africa [7]. To date, only one genotype 7 infection has been reported, and it was linked to a Central African immigrant in Canada $[5,6,9,10]$.

In Saudi Arabia, between 100,000 and 110,000 individuals are infected with CHC [11]. Genotype 4, followed by genotype 1, is considered more prevalent than other genotypes in Saudi Arabia, while genotype 3 and genotype 6 are found in extremely rare cases [12]. The Ministry of Health (MOH) in Saudi Arabia stated that 1,327 new cases of Hepatitis C were identified in 2015 with an incidence rate of 4.21 per 100,000 population [13]. Although it is hard to measure hepatitis C virus prevalence in Saudi Arabia, blood testing and screening suggest a prevalence rate of $0.4 \%-1.1 \%$ of the total population $[14,15]$. Linkage to care and treatment is critical in improving health for individuals found to be infected with hepatitis $C$ virus. Such linkage is important considering major developments in hepatitis $C$ virus treatments. With these developments, the epidemiology of the disease could differ within the upcoming years.

Over the last 25 years, hepatitis $C$ virus treatments developed from the first approved treatment alfa interferon (INF- $\alpha$ ) in 1991, which had a poor sustained virologic response (SVR) to the scientific breakthrough of the Direct Acting Antivirals (DAAs) [14]. Pegylated-interferon was developed and approved in 2001 for HCV treatment in combination with ribavirin [16]. Boceprevir and Telaprevir were the first-generation DAAs approved for HCV treatment in 2011, but they were discontinued within two years consequently of second-generation DAAs approval $[14,17,18]$. These second-generation DAAs shows a promising future in eradicating this virus infection. Thus they are now the standard-of-care for hepatitis $\mathrm{C}$ virus treatment in Saudi Arabia recommended by the Saudi Association for the Study of Liver Diseases and Transplantation (SASLT) [12,14]. These drugs in Saudi Arabia cost between $\$ 58,000$ - $\$ 67,000$ for 12 weeks of treatment in retail pharmacies based on Saudi Food and Drug Authority's (SFDA) price regulations $[18,19]$. The SFDA was established in 2003 as an Independent body corporate to help the $\mathrm{MOH}$ in their responsibilities and take over food, drug, and medical devices regulations.

In recent years, pharmacoeconomic evaluation has been introduced to healthcare technology assessment organizations expanding the spectrum of economic evaluations and outcomes research. Pharmacoeconomics is defined as the branch of economic evaluations that deals with pharmaceutical products and different disease management strategies [22]. The aim of pharmacoeconomic evaluation is to allocate health resources to obtain optimum value for patients and healthcare organizations [22,13].

The total healthcare expenditure associated with chronic hepatitis C in Saudi Arabia is difficult to determine due to lack of literature. In 2015, the Saudi Arabian government spent 7.25\% (\$16.5 billion) of total expenditures on health [14]. With the new recommendations to prioritize the new DAAs as first-line therapy in the treatment of chronic hepatitis $C$, it became a huge burden on every country, considering the high cost of treatments. Furthermore, 
adding the cost of preventing and monitoring chronic hepatitis $\mathrm{C}$ and its complications (such as liver cirrhosis, fibrosis, or cancer), extending the treatment in experience-patients, or co-infection with other disease states, the economic burden on governments and health organizations is continuing to grow. In 2014, there were 198 liver transplants in Saudi Arabia reported by the Saudi Center for Organ Transplantation (SCOT) with a total cost of $\$ 18,386,000$, the expert panel estimated that $45 \%$ of total liver transplantation in Saudi Arabia is attributed to HCV [26,27]. Thus, chronic hepatitis $C$ is a costly disease to manage for all managed care organizations which require economic allocation of resources toward medication selection and purchases to avoid huge budget impacts [28]. Although health care system in Saudi Arabia is provided and monitored by the Ministry of Health $(\mathrm{MOH})$, drug registration is regulated and monitored by the drug sector in the Saudi Food and Drug Authority (SFDA) while branded drugs' patency is obtained from King Abdulaziz city for science and technology (KACST) [2931]. Patients in Saudi Arabia prefer branded drugs over generics and can purchase these expensive drugs from retail pharmacies without a prescription [24]. With the treatments' high prices and new DAAs developed, more pharmacoeconomic studies should be considered before giving recommendations by government agencies and hospital's formulary committees in Saudi Arabia. The determinant of treatment guidelines in Saudi Arabia is solely based on clinical experience and expert judgment. Although pharmacoeconomic studies are helpful, decisions should not be based solely on them. The use of pharmacoeconomic analyses takes into consideration the clinical and economical perspectives. Therefore, it will be helpful and more efficient for improved judgment and budget control rather than relying on educated guesses or one-sided perception. Saudi Arabia is currently experiencing a new economic phase where pharmacoeconomic studies will be considered greatly [32-35]. Additionally, a study estimated that 200,000 hepatitis C Saudi patients would develop cirrhosis within the next 20 years, of them 1,500 new cases of hepatocellular carcinoma each year [37].

Due to the lack of pharmacoeconomic studies in Saudi Arabia, this study attempts to fill this gap and add a pharmacoeconomic perspective for decision makers in the government or hospitals regarding budget control and disease guidelines. Considering the benefits of these studies could lead to conducting further studies on different diseases in Saudi Arabia. Also, filling the gap in current literature will encourage future studies in the pharmacoeconomic field.

\section{Objective of the Study}

The study's objectives are (1) to estimate the total costs of hepatitis C treatment choices in Saudi Arabia based on data from the Saudi Food and Drug Authority (SFDA), (2) to develop and operationalize the decision tree model and calculate the base case incremental cost effectiveness ratio (ICER), (3) to perform one-way and probabilistic sensitivity analyses testing the underlying assumptions in the decision tree model.

\section{Methods}

A cost-effectiveness analysis was performed on a hypothetical cohort comparing different chronic hepatitis $\mathrm{C}$ treatment strategies from the (SFDA)'s perspective over a three-month period using a decision tree model. The decision tree model was developed using TreeAge Pro software [38]. Data for this study were obtained retrospectively from the (SFDA) and published literature. Costs were measured in United States Dollars (USD). Life-years gained (Ly) were the outcomes measured in this study. The data was implemented in the decision tree model following a hypothetical cohort to obtain the ICER's. Since the SASLT guidelines differ between genotype 1 and genotype 4, There were two separate decision tree models and analyses for each genotype cohort.

The base case population in this study was 40 years old Saudi Arabian patients diagnosed with chronic hepatitis $C$ genotype 1 or genotype 4 who were treatment-naïve (didn't receive treatment for chronic hepatitis $\mathrm{C}$ before), non-cirrhotic, not diagnosed with liver cancer, and didn't have a liver transplant or co-infections. Treatment interventions included in this study were based on the SASLT recommendation's (grade A1 and B1) and data availability in published literature. For genotype 1, four treatments were included: Ledipasvir/Sofosbuvir (grade A1), Sofosbuvir plus Simeprevir (grade A1), Elbasvir/Grazoprevir (grade A1), and Ritonavir/Paritaprevir/Ombitasvir and Dasabuvir with or without Ribavirin (grade A1). For genotype 4, three treatments were included: Ritonavir/ Paritaprevir/Ombitasvir with Ribavirin (grade A1), Sofosbuvir plus Simeprevir (grade B1), and Ledipasvir/Sofosbuvir (grade B1).

Decision tree model is an illustrative representation or mapping of all possible outcomes of a certain health state or intervention strategy in chronological order [39]. Each pathway in the decision tree is assigned a specific outcome and cost values. The effectiveness value of each pathway is calculated by multiplying the proba- 
bility of the pathway with their respective outcome value. The cost for each pathway is cumulatively aggregated at the terminal node where the incremental cost-effectiveness ratio (ICER) is calculated with the alternative intervention strategies. The model was developed using TreeAge Pro software 2015, R1.0, Williamstown, MA. The model simulated the progression of non-cirrhotic chronic hepatitis $\mathrm{C}$ cohort who were under different treatment alternatives. The model followed the hypothetical cohort for three months. In genotype 1 model, the cohort was initiated on one of the four treatments while in genotype 4 the cohort was initiated on one of the three treatments. Side effects of each treatment were integrated into the model. Cost, Outcomes, and side effects of the treatments were assigned for each pathway in the model. Probabilities for the treatments cure rate and side effects were assigned for each pathway. After creating the models, they were examined and validated by clinical and pharmacoeconomic expertise. The data was imple- mented for each cohort model to run the analysis and generate the base case scenario and obtain the incremental cost-effectiveness ratio (ICER). The decision tree model for each genotype cohort is shown in figure 1 and 2.

Only direct medical costs were included from the perspective of the Saudi Food and Drug Authority (SFDA). The costs included were drug costs and side effects treatment costs (anemia, rash, depression). Any costs assumed to be applied to every patient in the cohort were opt out from the analysis (e.g. laboratory tests cost). The cost of Ribavirin and Sofosbuvir were analyzed in sensitivity analysis since they had generic drugs registered. Costs for side effects' treatments were calculated based on the average common prescription drug costs used to treat it. All costs were obtained from the SFDA [20]. All costs were converted to 2017 United States Dollars. All costs included in the base case analyses are shown in table 1.

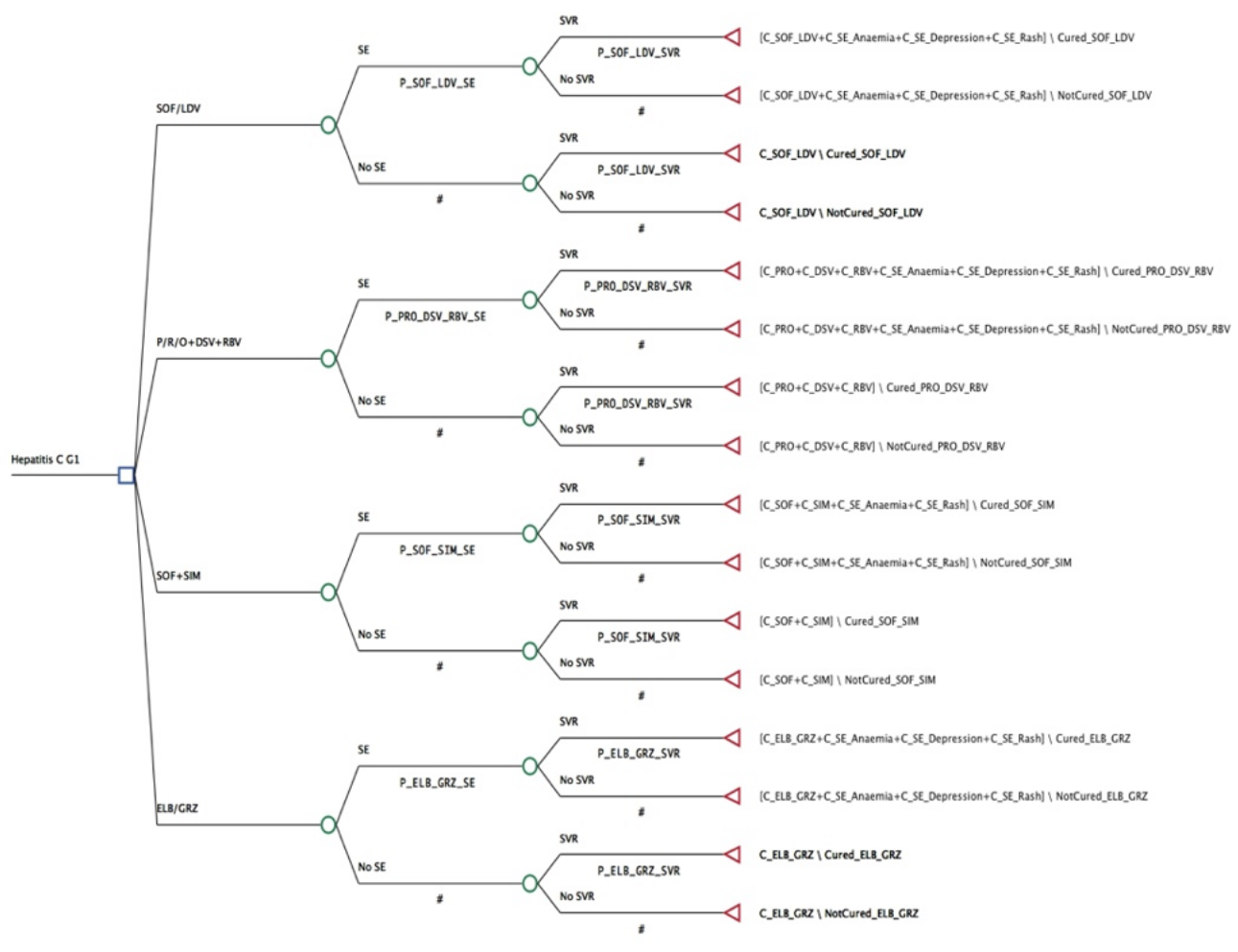

Figure 1: Decision tree model for chronic hepatitis C genotype 1 build in TreeAge Pro software. 


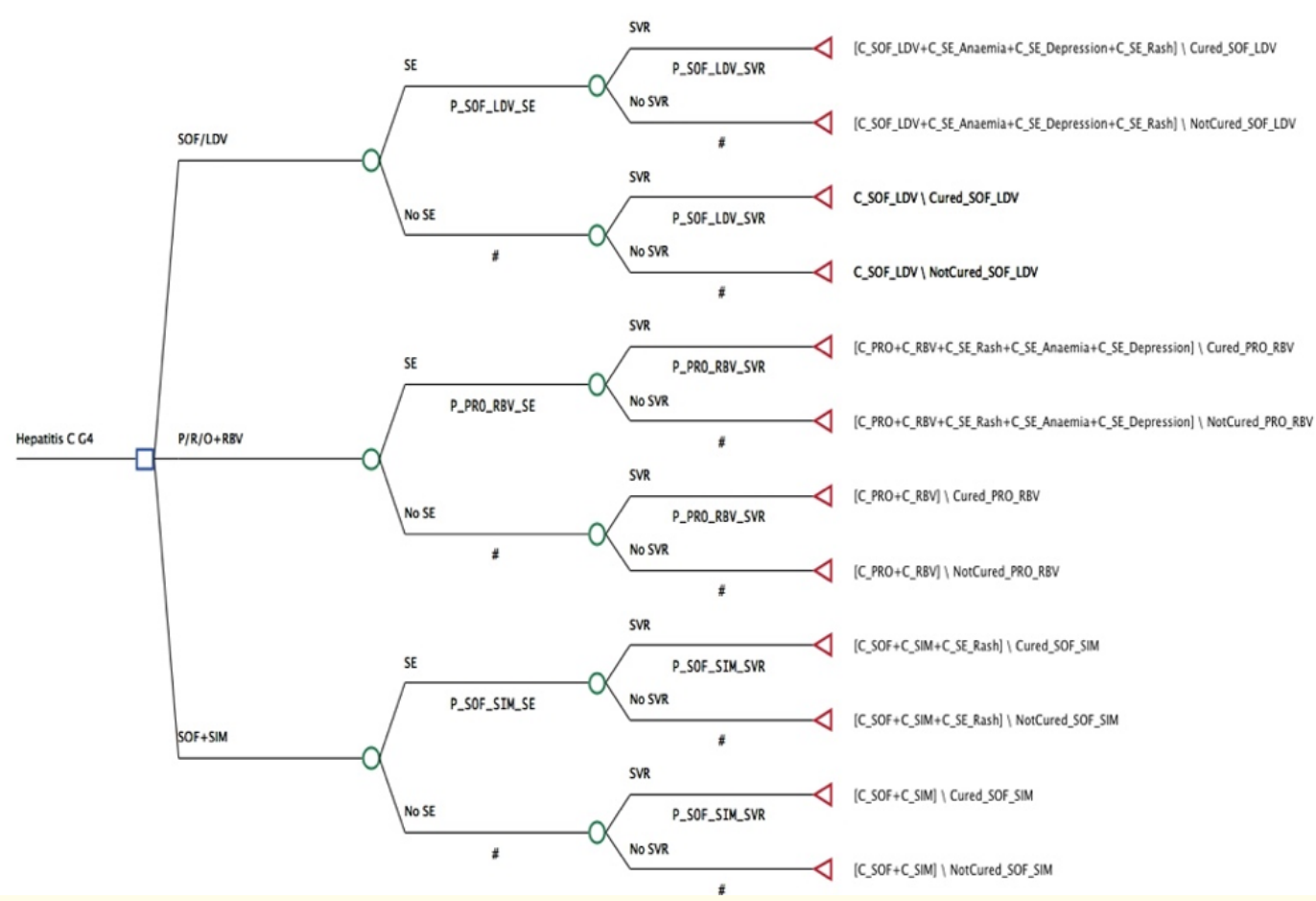

Figure 2: Decision tree model for chronic hepatitis C genotype 4 build in TreeAge Pro software.

\begin{tabular}{|l|c|}
\hline \multicolumn{1}{|c|}{ Variable } & Cost (\$) \\
\hline Ledipasvir/Sofosbuvir & $66,446.48$ \\
\hline Sofosbuvir & $49,647.06$ \\
\hline Simeprevir & $37,680.16$ \\
\hline Ritonavir/Paritaprevir/Ombitasvir & $65,171.00$ \\
\hline Dasabuvir & $5,151.84$ \\
\hline Ribavirin & 0.00 \\
\hline Elbasvir/Grazoprevir & $58,054.60$ \\
\hline Depression treatment & 160.96 \\
\hline Rash treatment & 94.34 \\
\hline Anemia treatment & $1,176.56$ \\
\hline
\end{tabular}

Table 1: Cost inputs into the decision tree models from the SFDA.

The Outcome measured in this study is life-years gained (Ly) derived by the cure rate of each treatment intervention. The cure rate for each treatment is expressed as sustained virologic response (SVR) which represents the eradication of hepatitis $C$ virus from the patient's system. After completing the treatment course of three months, patients with SVR were considered cured. From published literature on Saudi Arabian population, the average patient's age of chronic hepatitis $C$ diagnosis was 40 years old $[27,40]$. Based on the World Health Organization (WHO), the life expectancy of Saudi Arabian population is 75 years [41]. By subtracting the average age of patient's diagnosis from the population's life expectancy, we find that the life-years gained form each successful treatment (complete cure) is 35 years. In the model, we assigned each successful treatment with 35 years (represented in the model as cured) and every treatment failure with 0 years (represented in the model as not cured). Not gaining any life-years did not mean death, but it represented that the treatment failed to cure the patient; therefore, the patient did not gain any life-years consequent to the treatment (i.e. the treatment was not effective). For each genotype cohort in this study, the probability of each intervention's successful cure and side effects were calculated based on pooled data from clinical trials (LONESTAR, C-WORTHY, COSMOS, ION-I, IONII, ION-III, PLUTO, PEARL-III, PEARL-IV) and published literature $[12,33,34,42-54]$. Treatments' cure rates (probabilities) were mea- 
sured by the ability of the patient to reach a sustained virologic response (SVR) within three months of treatment administration. Due to clinical trials combining results for genotype $1 \mathrm{a}$ and $1 \mathrm{~b}$, we assumed treatment outcomes and side effects were similar. The probabilities for each genotype decision tree model in this study are shown in table 2 . In genotype 1 base case analysis, Ribavirin cost were $\$ 0$ to allow combining genotype $1 \mathrm{a}$ and $1 \mathrm{~b}$ interventions into one analysis, assuming they had similar outcomes, and examine Ribavirin's cost in sensitivity analysis. The effectiveness measured was life-years gained (Ly). Cost was estimated in 2017 United States Dollars (USD). Probabilities were calculated based on pooled data from clinical trials and published literature. The time horizon for both analyses was three months; therefore, no annual discounting was performed. The perspective in this study was from a governmental body (the SFDA). The willingness to pay (WTP) were set to $\$ 65,000$ based on Saudi Arabia's GDP per capita $(\$ 21,000)$ [55]. After running the analyses, the base case ICER's were generated. Sensitivity analyses were then performed to test the robustness of each base case scenario. Uncertainties surrounding the base case scenarios were explored through deterministic sensitivity analysis (DSA) and probabilistic sensitivity analysis (PSA). Deterministic sensitivity analysis assesses uncertainties by setting low and high values for each input independently without changing the base case value inputs. The deterministic sensitivity analysis performed in this study examined the cost of Sofosbuvir and Ribavirin because they had generic alternatives registered in the SFDA. Probabilistic sensitivity analysis utilizes statistical distributions of parameter inputs to run multiple simulations. Distributions for costs, outcomes, and probabilities inputs in this study were used in the probabilistic sensitivity analyses to generate 1,000 Monte Carlo simulations for both models. Probabilities assumed to have beta distribution with standard deviation ranged between 0.01-0.05 as obtained from clinical trials for each intervention. The cost inputs of every intervention were varied by $30 \%$ on both ends. Acceptability curves for each genotype analysis were represented at a willingness to pay of $\$ 65,000$.

\section{Results}

\section{Effectiveness}

For genotype 1, Paritaprevir/Ritonavir/ombitasvir plus Dasabuvir with Ribavirin had the highest cumulative life-years gain of 34.18 years, followed by Ledipasvir/Sofosbuvir, Elbasvir/Grazoprevir, then Sofosbuvir plus Simeprevir with a cumulative life-

\begin{tabular}{|l|c|c|}
\hline \multicolumn{1}{|c|}{ Variable } & $\begin{array}{c}\text { G1 } \\
\text { Probability }\end{array}$ & $\begin{array}{c}\text { G4 } \\
\text { Probability }\end{array}$ \\
\hline Ledipasvir/Sofosbuvir SVR & 0.973 & 0.955 \\
\hline Ledipasvir/Sofosbuvir SE & 0.0267 & 0.00 \\
\hline Sofosbuvir + Simeprevir SVR & 0.9645 & 0.998 \\
\hline Sofosbuvir + Simeprevir SE & 0.0179 & 0.05 \\
\hline $\begin{array}{l}\text { Ritonavir/Paritaprevir/ } \\
\text { Ombitasvir with Ribavirin SVR }\end{array}$ & $\begin{array}{c}0.9766(\text { with } \\
\text { Dasabuvir) }\end{array}$ & 0.998 \\
\hline $\begin{array}{l}\text { Ritonavir/Paritaprevir/ } \\
\text { Ombitasvir with Ribavirin SE }\end{array}$ & $\begin{array}{c}0.0456(\text { with } \\
\text { Dasabuvir) }\end{array}$ & 0.036 \\
\hline Elbasvir/Grazoprevir SVR & 0.969 & N/A \\
\hline Elbasvir/Grazoprevir SE & 0.0368 & N/A \\
\hline
\end{tabular}

Table 2: Probabilities input of chronic hepatitis C genotype 1and genotype 4 into the decision tree model.

years gain of 34.07, 33.91, and 33.76 years respectively. For genotype 4, both Paritaprevir/Ritonavir/Ombitasvir with Ribavirin and Sofosbuvir plus Simeprevir had the highest cumulative life-years gain of 34.96 years followed by Ledipasvir/Sofosbuvir with a lifeyears gain of 33.42 years.

Cost

In genotype 1, Elbasvir/Grazoprevir was associated with the lowest cumulative cost of $\$ 58,107$. Ledipasvir/Sofosbuvir, Paritaprevir/Ritonavir/Ombitasvir and Dasabuvir with Ribavirin, and Sofosbuvir plus Simeprevir had cumulative costs of $\$ 66,485$, $\$ 70,388$, and $\$ 87,350$, respectively. In genotype 4 , the lowest cumulative cost was for Paritaprevir/Ritonavir/Ombitasvir with Ribavirin intervention of $\$ 65,223$, followed by Ledipasvir/Sofosbuvir and Sofosbuvir plus Simeprevir with cumulative costs of $\$ 66,446$ and $\$ 87,332$, respectively.

\section{Base case analysis}

In genotype 1 base case analysis, the cost-effectiveness ratio (cost per $1 \mathrm{Ly}$ ) of the interventions were $\$ 1,713$ per Ly for Elbasvir/Grazoprevir, \$1,952 per Ly for Sofosbuvir/Ledipasvir, \$2,059 per Ly for Paritaprevir/Ritonavir/Ombitasvir and Dasabuvir with Ribavirin, and \$2,588 per Ly for Sofosbuvir plus Simeprevir. The incremental cost-effectiveness comparison between the interventions showed that both Elbasvir/Grazoprevir and Paritaprevir/ Ritonavir/Ombitasvir plus Dasabuvir with Ribavirin were cost-effective at WTP of $\$ 65,000$. Elbasvir/Grazoprevir showed absolute dominance on Sofosbuvir with Simeprevir and extended domi- 
nance on Ledipasvir/Sofosbuvir. Against Paritaprevir/Ritonavir/ Ombitasvir and Dasabuvir with Ribavirin, the ICER was $\$ 33,796$ / Ly for each additional cure. In genotype 4 base case analysis, the cost-effectiveness ratio (cost per $1 \mathrm{Ly}$ ) of the interventions were $\$ 1,865$ per Ly for Paritaprevir/Ritonavir/Ombitasvir with Ribavirin, \$1,988 per Ly for Ledipasvir/Sofosbuvir, and \$2,498 per Ly for Sofosbuvir plus Simeprevir. The incremental cost-effectiveness comparison between the interventions showed that Paritaprevir/ Ritonavir/Ombitasvir with Ribavirin was cost-effective at WTP of $\$ 65,000$. Paritaprevir/Ritonavir/Ombitasvir with Ribavirin showed absolute dominance on Ledipasvir/Sofosbuvir and Sofosbuvir plus Simeprevir. Results of base case scenarios are shown in table 3 and 4.

\begin{tabular}{|l|c|c|c|c|}
\hline \multicolumn{1}{|c|}{ Intervention } & $\begin{array}{c}\text { Total } \\
\text { Cost(\$) }\end{array}$ & $\begin{array}{c}\text { Ly } \\
\text { (Years) }\end{array}$ & $\begin{array}{c}\text { Cost/1 Ly } \\
\text { (\$/Ly) }\end{array}$ & $\begin{array}{c}\text { ICER } \\
\text { (\$/Ly) }\end{array}$ \\
\hline $\begin{array}{l}\text { Elbasvir/ } \\
\text { Grazoprevir }\end{array}$ & 58,107 & 33.91 & 1,714 & $\begin{array}{c}\text { Refer- } \\
\text { ence }\end{array}$ \\
\hline $\begin{array}{l}\text { Paritaprevir/ } \\
\text { Ritonavir/ } \\
\text { Ombitasvir+ Das- } \\
\text { abuvir + Ribavirin }\end{array}$ & 70,388 & 34.18 & 2,059 & 33,796 \\
\hline $\begin{array}{l}\text { Ledipasvir/Sofos- } \\
\text { buvir }\end{array}$ & 66,485 & 34.07 & 1,952 & $53,190^{*}$ \\
\hline $\begin{array}{l}\text { Sofosbuvir + } \\
\text { Simeprevir }\end{array}$ & 87,350 & 33.76 & 2,588 & $-40,052^{* *}$ \\
\hline
\end{tabular}

Table 3: Results from genotype 1 cohort base case scenario.

*: The intervention was dominated, **: The intervention was dominated; negative sign due to the intervention being less effective and more expensive

\begin{tabular}{|l|c|c|c|c|}
\hline \multicolumn{1}{|c|}{ Intervention } & $\begin{array}{c}\text { Total } \\
\text { Cost (\$) }\end{array}$ & $\begin{array}{c}\text { Ly } \\
\text { (Years) }\end{array}$ & $\begin{array}{c}\text { Cost/1 } \\
\text { Ly (\$/Ly) }\end{array}$ & $\begin{array}{c}\text { ICER (\$/ } \\
\text { Ly) }\end{array}$ \\
\hline $\begin{array}{l}\text { Paritaprevir/Ritonavir/ } \\
\text { Ombitasvir +Ribavirin }\end{array}$ & 65,223 & 34.96 & 1,865 & $\begin{array}{c}\text { Refer- } \\
\text { ence }\end{array}$ \\
\hline Ledipasvir/Sofosbuvir & 66,446 & 33.42 & 1,988 & $-795^{*}$ \\
\hline Sofosbuvir + Simeprevir & 87,332 & 34.96 & 2,498 & $0^{* *}$ \\
\hline
\end{tabular}

Table 4: Results from genotype 4 cohort base case scenario.

*: The intervention was dominated; negative sign due to the intervention being less effective and more expensive, ${ }^{* *}$ : Null ICER due to intervention's equal effectiveness and increased cost to comparator.
Sensitivity analyses

Deterministic (One-way) and probabilistic sensitivity analyses were performed on the uncertainties surrounding the base case scenario of genotype 1 model inputs. Individually, the costs of Sofosbuvir and Ribavirin were altered while keeping all other parameters exactly as the base case input values. Ribavirin and Sofosbuvir generics' costs registered in the SFDA was $\$ 627.5$ and $\$ 4,480$, respectively. After running the one-way sensitivity analyses at a $\$ 65,000$ WTP, the cost of Ribavirin showed no effect on the robustness of the base case scenario. Varying Sofosbuvir's cost resulted in Sofosbuvir plus Simeprevir and Paritaprevir/Ritonavir/ Ombitasvir plus Dasabuvir with Ribavirin being cost-effective with an ICER of $\$ 33,796 /$ Ly for each additional patient on Paritaprevir/ Ritonavir/Ombitasvir plus Dasabuvir with Ribavirin. In the Probabilistic sensitivity analysis, 1,000 simulations using different parameter inputs in the base case analysis simultaneously at different WTP thresholds are shown in the acceptability curve for genotype 1 analysis (Figure 3). At $\$ 65,000$ WTP, Ledipasvir/Sofosbuvir was cost effective in $27 \%$ of all cases followed by Paritaprevir/Ritonavir/Ombitasvir plus Dasabuvir with Ribavirin (26\% of all cases) and Elbasvir/Grazoprevir (24.5\% of all cases). The frequency of being the optimal intervention (cost effective) at $\$ 65,000 \mathrm{WTP}$ are shown in figure 4 . In genotype 4 deterministic (One-way) and probabilistic sensitivity analyses at a $\$ 65,000 \mathrm{WTP}$, the cost of Ribavirin showed no effect on the robustness of the base case scenario while varying Sofosbuvir's cost resulted in Sofosbuvir plus Simeprevir dominating other interventions at a total cost of $\$ 42,165$. Genotype 4 acceptability curve yield Paritaprevir/Ritonavir/Ombitasvir with Ribavirin was cost-effective (Figure 5). At \$65,000 WTP, Paritaprevir/Ritonavir/Ombitasvir with Ribavirin dominated $37.8 \%$ of all cases followed by Sofosbuvir plus Simeprevir in 36\% of all cases (Figure 6).

\section{Discussion}

The study addresses the economic evaluation of genotype 1 and genotype 4 treatment alternatives in non-cirrhotic Saudi Arabian patients utilizing data from the SFDA, clinical trials, and published literature. In the base-case analyses, Elbasvir/Grazoprevir in genotype 1 and Paritaprevir/Ritonavir/Ombitasvir based regimen in both genotypes were cost-effective at a WTP of \$65,000/Ly. In genotype 1, Ledipasvir/Sofosbuvir showed slight increments in cost and efficacy than Elbasvir/Grazoprevir, but Paritaprevir/Ritona- 


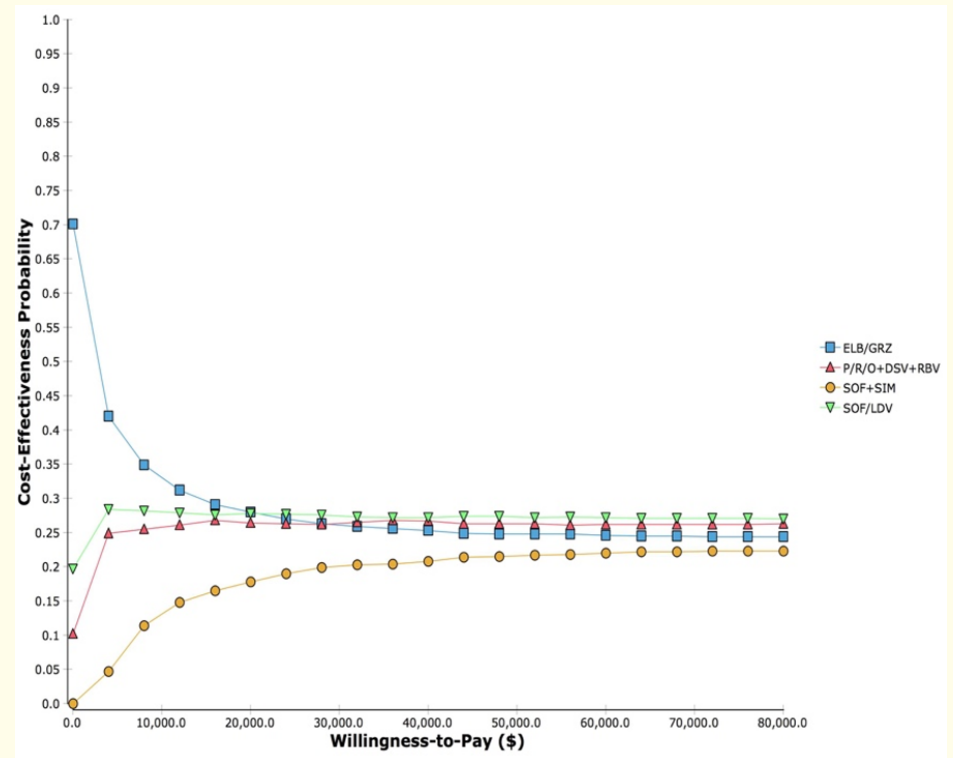

Figure 3: Cost-effectiveness acceptability curve showing the cost-effectiveness probability at the willingness-to-pay in genotype 1 probabilistic sensitivity analysis.

Willingness-to-pay in US dollars, ELB/GRZ: Elbasvir/Grazoprevir, P/R/O+DSV+RBV: Paritaprevir/Ritonavir/

Ombitasvir + Dasabuvir + Ribavirin, SOF+SIM: Sofosbuvir + Simeprevir, SOF/LDV: Ledipasvir/Sofosbuvir.

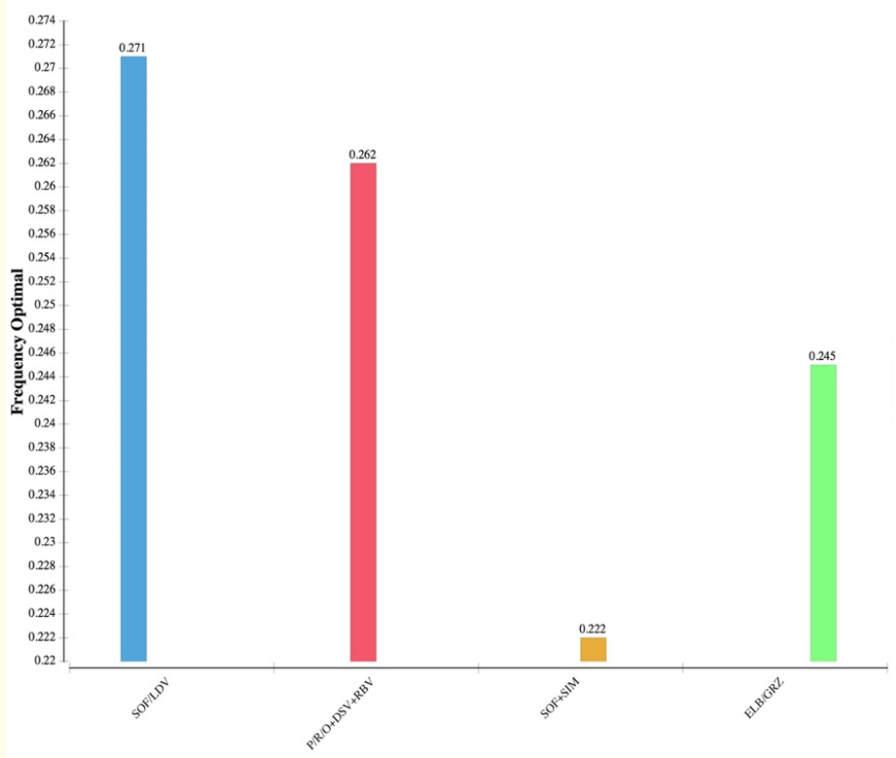

Figure 4: Frequency distribution of optimal intervention at $\$ 65,000$ willingness-to-pay in genotype 1 probabilistic sensitivity analysis. ELB/GRZ: Elbasvir/Grazoprevir, P/R/O+DSV+RBV: Paritaprevir/Ritonavir/Ombitasvir + Dasabuvir + Ribavirin, SOF+SIM: Sofosbuvir + Simeprevir, SOF/LDV: Ledipasvir/Sofosbuvir. 


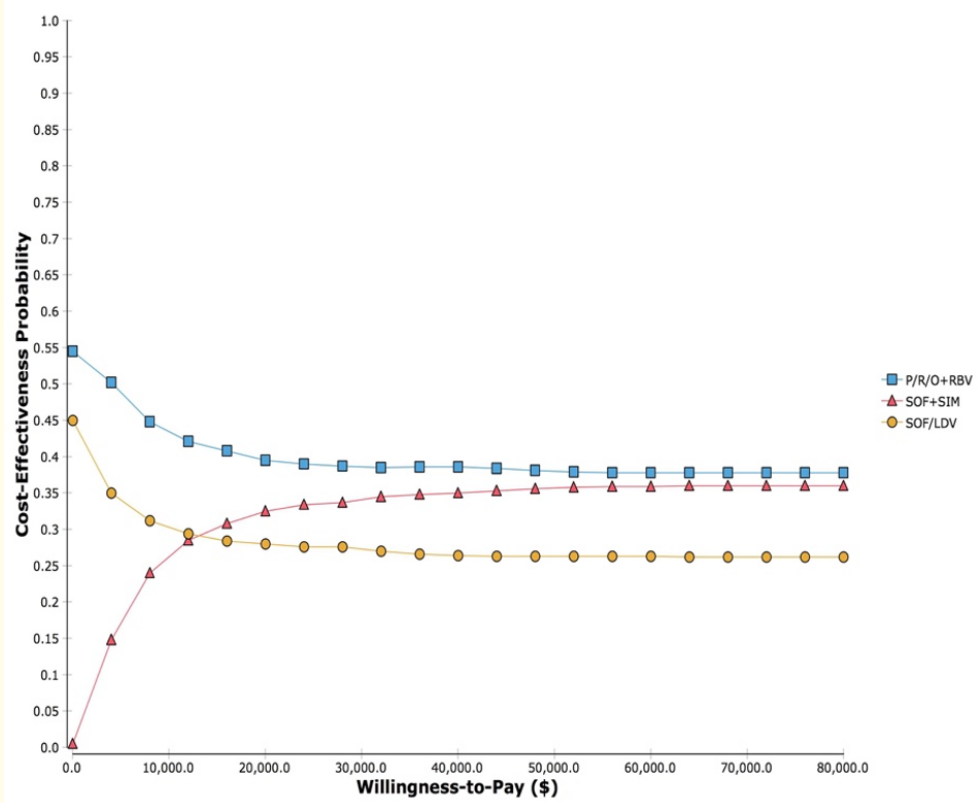

Figure 5: Cost-effectiveness acceptability curve showing the cost-effectiveness probability at the willingness-to-pay in genotype 4 probabilistic sensitivity analysis.

Willingness-to-pay in US dollars, P/R/O+RBV: Paritaprevir/Ritonavir/Ombitasvir + Ribavirin, SOF+SIM:

Sofosbuvir + Simeprevir, SOF/LDV: Ledipasvir/Sofosbuvir.

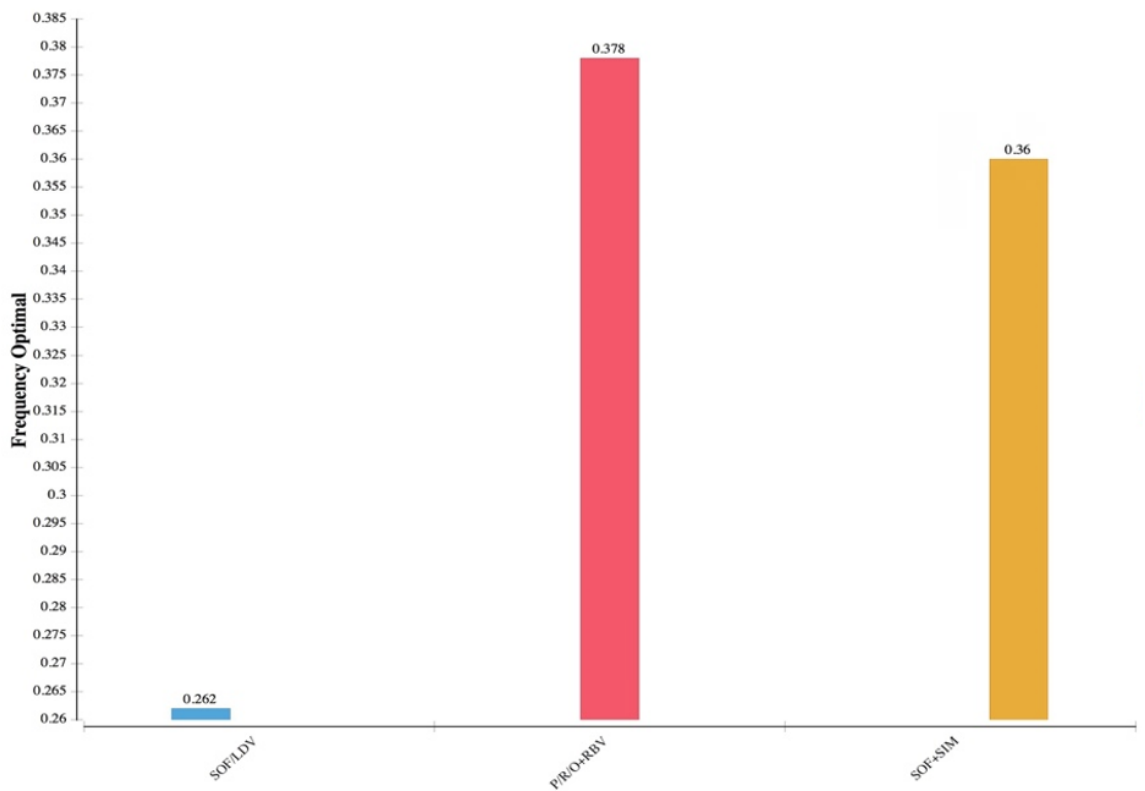

Figure 6: Frequency distribution of optimal intervention at $\$ 65,000$ willingness-to-pay in genotype 4 probabilistic sensitivity analysis. P/R/O+RBV: Paritaprevir/Ritonavir/Ombitasvir + Ribavirin, SOF+SIM: Sofosbuvir + Simeprevir, SOF/LDV: Ledipasvir/Sofosbuvir. 
vir/Ombitasvir plus Dasabuvir with Ribavirin had higher cost-effectiveness profile than Ledipasvir/Sofosbuvir. Thus, Ledipasvir/ Sofosbuvir were dominated by the presence of both interventions in the cohort treatment. In the absence of Elbasvir/Grazoprevir clinical data on genotype 4 patients' treatment, Paritaprevir/Ritonavir/Ombitasvir with Ribavirin dominated other comparators. The study findings were similar to a recent study done in the United States that showed Paritaprevir/Ritonavir/Ombitasvir based regimens cost-effective [36]. Saab., et al. represented the treatment to be associated with lower cost-effectiveness ratios in both cohorts, which is not the case in this study. However, most of the published studies within the last years involved comparisons of DAAs with old regimens (INF-based); hence the interventions were presumed cost-effective [36]. In contrast, this study analyzed second-generation DAAs with high leveled quality evidence. Also, few clinical trials were done on genotype 4 second-generation DAAs which designated the assumptions in most studies to simulate genotype 4 to genotype 1 due to high relevance. Thus, numerous uncertainties found in pharmacoeconomic studies done on genotype 4 .

\section{Limitations}

There are some limitations to this study and the model used due to lack of data on Saudi population including; adherence or patient medication preferences, cirrhosis or other hepatitis C complications, limited interventions side effects. With access to real world data on Saudi Arabian patients, these limitations could be overcome.

\section{Conclusion}

The interventions compared in genotype 1 are competitive and cost effective in exception of Sofosbuvir with Simeprevir while $2 \mathrm{D}+\mathrm{RBV}$ is highly recommended in genotype 4 . Interventions in both genotypes will be dominated by Sofosbuvir low-priced generics.

\section{Conflict of Interests}

The listed authors had no potential conflict of interest, any affiliation, or a financial interest in the results of the study.

\section{Financial Disclosure}

This study did not receive any funding or grant.

\section{Acknowledgement}

Software: TreeAge Pro software 2015, R1.0, Williamstown, MA. Was used in this study.

\section{Bibliography}

1. WHO. "Hepatitis C". World Health Organization (2016).

2. Wang LS., et al. "Hepatitis C-A clinical review". Journal of Medical Virology 88 (2016): 1844-1855.

3. CDC. Surveillance for viral hepatitis - United States, 2013 (2016).

4. AASLD/IDSA. Recommendations for testing, managing, and treating hepatitis $\mathrm{C}$.

5. Thomas XV. "Epidemiological, immunological and virological aspects of acute and chronic hepatitis C virus infections" (2015).

6. Murphy DG., et al. "Use of sequence analysis of the NS5B region for routine genotyping of hepatitis $\mathrm{C}$ virus with reference to $\mathrm{C} /$ E1 and 5' untranslated region sequences". Journal of Clinical Microbiology 45 (2007): 1102-1112.

7. Shire NJ and Sherman KE. "Epidemiology of Hepatitis C Virus: A Battle on New Frontiers". Gastroenterology clinics of North America 44.4 (2015): 699-716.

8. Messina JP., et al. "Global distribution and prevalence of hepatitis C virus genotypes”. Hepatology 61.1 (2015): 77-87.

9. Shepard CW., et al. "Global epidemiology of hepatitis C virus infection". The Lancet Infectious Diseases 5.9 (2005): 558-567.

10. Simmonds P. "The origin and evolution of hepatitis viruses in humans". Journal of General Virology 82 (2001): 693-712.

11. Mohamed AA., et al. "Hepatitis C virus: A global view". World Journal of Hepatology 7.26 (2015): 2676.

12. Alghamdi AS., et al. "SASLT Position Statement on the DirectActing Antiviral Agents for the Treatment of Hepatitis C Virus Infection". Saudi Journal of Gastroenterology: Official Journal of the Saudi Gastroenterology Association 21.2 (2015): 60-63. 
13. Team MP. Kingdom of Saudi Arabia - ministry of health portal (2015).

14. Alghamdi AS., et al. "SASLT Guidelines: Update in Treatment of Hepatitis C Virus Infection". Saudi Journal of Gastroenterology: Official Journal of the Saudi Gastroenterology Association 22 (2015): S25-S57.

15. Abdo AA., et al. "Epidemiology of Viral Hepatitis in Saudi Arabia: Are We Off the Hook?" Saudi Journal of Gastroenterology: Official Journal of the Saudi Gastroenterology Association 18.6 (2012): 349-357.

16. Drugs@FDA: FDA approved drug products (2016).

17. Zhang X. "Direct anti-HCV agents". Acta Pharmaceutica Sinica B 6.1 (2016): 26-31.

18. Saudi Food and Drug Authority - drug list search (2016).

19. Saudi Food and Drug Authority Regulations and guidelines (2016).

20. Noureddin M., et al. "Association of IL28B genotype with fibrosis progression and clinical outcomes in patients with chronic hepatitis C: A longitudinal analysis". Hepatology 58.5 (2013): 1548-1557.

21. Boglione L., et al. "The Role of IL28B Genotype in HCV-RNA Baseline Levels". Intervirology 59.2 (2016): 67-68.

22. Arenas-Guzman R., et al. "Pharmacoeconomics-an aid to better decision-making". Journal of the European Academy of Dermatology and Venereology 19 (2005): 34-39.

23. Trask LS. Pharmacoeconomics: Principles, Methods, and Applications: Pharmacotherapy: A Pathophysiologic approach (2011).

24. Al-Jazairi AS., et al. "Pharmacoeconomic Analysis in Saudi Arabia: An Overdue Agenda Item for Action”. Annals of Saudi Medicine 31.4 (2011): 335-341.

25. Marseille E., et al. "Thresholds for the cost-effectiveness of interventions: alternative approaches". Bulletin of the World Health Organization 93.2 (2015): 118-124.

26. Saudi Center for Organ Transplantation (2016).
27. Aljumah AA., et al. "Epidemiology, Disease Burden, and Treatment Strategies of Chronic Hepatitis C Virus Infections in Saudi Arabia in the New Treatment Paradigm Shift". Saudi Journal of Gastroenterology: Official Journal of the Saudi Gastroenterology Association 22.4 (2016): 269-281.

28. Davis KL., et al. "Direct economic burden of chronic hepatitis C virus in a United States managed care population". Journal of Clinical Gastroenterology 45 (2011): e17-e24.

29. Alkhamis A. "Health care system in Saudi Arabia: an overview". Eastern Mediterranean Health Journal 18.10 (2012): 10781080

30. Saudi Arabia: Saudi Food and Drug Authority - SFDA (2017).

31. SPO Regulations. (KACST) (2017).

32. Saudi Arabian Monetary Agency Yearly statistics (2016).

33. Devarajan, Shantayanan Mottaghi Lili. MENA Quarterly Economic Brief, July 2016: Whither Oil Prices?. Washington, DC: World Bank. (C) World Bank (2016).

34. FocusEconomics. Saudi Arabia economy - GDP, inflation, CPI and interest rate (2016).

35. Ministry of Economy and Planning. Knowledge resources ministry of economy and planning (2016).

36. Saab S., et al. "Cost-effectiveness of currently recommended direct-acting antiviral treatments in patients infected with genotypes 1 or 4 hepatitis C virus in the US". Journal of Medical Economics (2016): 1-11.

37. Liu S., et al. "New protease inhibitors for the treatment of chronic hepatitis C: a cost-effectiveness analysis". Annals of Internal Medicine 156.4 (2012): 279-290.

38. TreeAge Pro. R1.0. TreeAge Software, Williamstown, MA software (2015).

39. Ryder HF, et al. "Decision Analysis and Cost-effectiveness Analysis”. Seminars in Spine Surgery 21.4 (2009): 216-222.

40. Liakina V., et al. "Historical epidemiology of hepatitis $\mathrm{C}$ virus (HCV) in select countries-volume 3". Journal of viral hepatitis 22 (2015): 4-20. 
41. Saudi Arabia, World Health Organization (2017).

42. Lawitz E., et al. "Sofosbuvir and ledipasvir fixed-dose combination with and without ribavirin in treatment-naive and previously treated patients with genotype 1 hepatitis $C$ virus infection (LONESTAR): an open-label, randomised, phase 2 trial". The Lancet 383.9916 (2014): 515-523.

43. Lawitz E., et al. "Efficacy and safety of 12 weeks versus 18 weeks of treatment with grazoprevir (MK-5172) and elbasvir (MK-8742) with or without ribavirin for hepatitis $C$ virus genotype 1 infection in previously untreated patients with cirrhosis and patients with previous null response with or without cirrhosis (C-WORTHY): a randomised, open-label phase 2 trial". The Lancet 385.9973 (2015): 1075-1086.

44. Lawitz E., et al. "Simeprevir plus sofosbuvir, with or without ribavirin, to treat chronic infection with hepatitis $C$ virus genotype 1 in non-responders to pegylated interferon and ribavirin and treatment-naive patients: the COSMOS randomised study". The Lancet 384.9956 (2014): 1756-1765.

45. Lawitz E., et al. "Efficacy and safety of ombitasvir, paritaprevir, and ritonavir in an open-label study of patients with genotype $1 \mathrm{~b}$ chronic hepatitis $\mathrm{C}$ virus infection with and without cirrhosis". Gastroenterology 149.4 (2015): 971-980.

46. Younossi ZM., et al. "Ledipasvir/sofosbuvir treatment of hepatitis $C$ virus is associated with reduction in serum apolipoprotein levels". Journal of Viral Hepatitis 22.12 (2015): 977-982.

47. Younossi, ZM., et al. "Treatment with ledipasvir and sofosbuvir improves patient-reported outcomes: Results from the ION-1,-2, and-3 clinical trials". Hepatology 61.62 (2015): 1798-1808.

48. Abergel A., et al. "Ledipasvir plus sofosbuvir for 12 weeks in patients with hepatitis C genotype 4 infection". Hepatology 64.4 (2016): 1049-1056.

49. Kumada H., et al. "The combination of elbasvir and grazoprevir for the treatment of chronic HCV infection in Japanese patients: a randomized phase II/III study". Journal of Gastroenterology (2016): 1-14.
50. Zeuzem S., et al. "Grazoprevir-Elbasvir Combination Therapy for Treatment-Naive Cirrhotic and Noncirrhotic Patients With Chronic Hepatitis C Virus Genotype 1, 4, or 6 InfectionA Randomized TrialC-EDGE Treatment-Naive Trial of GrazoprevirElbasvir". Annals of Internal Medicine 163.1 (2015): 1-13.

51. Buti M., et al. "Simeprevir in combination with sofosbuvir in treatment-naïve and-experienced patients with hepatitis $\mathrm{C}$ virus genotype 4 infection: a Phase III, open-label, single-arm study (PLUTO)". Alimentary Pharmacology and Therapeutics 45.3 (2017): 468-475.

52. Ferenci P., et al. "ABT-450/r-ombitasvir and dasabuvir with or without ribavirin for HCV". New England Journal of Medicine 370.21 (2014): 1983-1992.

53. Andreone P., et al. "ABT-450, ritonavir, ombitasvir, and dasabuvir achieves $97 \%$ and $100 \%$ sustained virologic response with or without ribavirin in treatment-experienced patients with HCV genotype 1b infection". Gastroenterology 147.2 (2014): 359-365.

54. Feld JJ., et al. "Treatment of HCV with ABT-450/r-ombitasvir and dasabuvir with ribavirin". New England Journal of Medicine 370.17 (2014): 1594-1603.

55. Saudi Arabia GDP per capita | 1968-2017 | Data | Chart | Calendar.

56. Zhang S., et al. "Cost-effectiveness of sofosbuvir-based treatments for chronic hepatitis C in the US". BMC Gastroenterology 15.1 (2015): 98.

\section{Assets from publication with us}

- Prompt Acknowledgement after receiving the article

- Thorough Double blinded peer review

- Rapid Publication

- Issue of Publication Certificate

- High visibility of your Published work

Website: www.actascientific.com/

Submit Article: www.actascientific.com/submission.php

Email us: editor@actascientific.com

Contact us: +919182824667 